\title{
Pigmentation associated histopathological variations in sympathetic ophthalmia
}

\author{
GEORGE E. MARAK, JR., AND HIROSHI IKUI \\ From the Department of Ophthalmology, Georgetown University, Washington, DC, USA, and the \\ Department of Ophthalmology, Kyushu University, Fukuoka, Japan
}

SUMMARY The severity of inflammation in sympathetic ophthalmia is related to the degree of pigmentation, and the granulomatous response seems to be related to pigmentation. Eosinophilia is also associated with pigmentation, but this association appears to be fortuitous and is a result of the association of eosinophilia with severity of the inflammation.

Elsching presented his most confusing pearl to the ophthalmological community when he proposed that sympathetic ophthalmia represented an autoimmune response to uveal pigment. ${ }^{12}$ Recent observations have raised considerable doubts about the role of uveal pigment as the offending antigenin sympathetic ophthalmia. ${ }^{34}$ The function of the uveal melanocytes in sympathetic ophthalmia is not well understood, but pigmentary disturbances occur clinically, and some histopathological features of sympathetic ophthalmia appear to be associated with uveal pigmentation. ${ }^{5}$ The purpose of this report is to evaluate the previously described association of certain histopathological features of sympathetic ophthalmia with pigmentation in a new population.

\section{Materials and methods}

Cases were selected for this study from the files on sympathetic ophthalmia at Kyushu University, Fukuoka, Japan, and from the Armed Forces Institute of Pathology (AFIP), USA, that fulfilled well established clinical and histopathological criteria for the diagnosis of sympathetic ophthalmia outlined by Easom and Zimmerman. ${ }^{6}$

In the present study 2 groups of patients were selected who appeared to be intermediate in pigmentation between the black and white groups of patients described in a previous report. ${ }^{5}$ One group of patients were Japanese and the second group was a heterogeneous collection of Indian subcontinent, American Indian, Puerto Rican, Middle East, and non-Japanese oriental patients who might

Correspondence to George E. Marak, Jr., MD, 1451 Belle Haven Road, Alexandria, Virginia 22307, USA. reasonably be considered intermediate in pigmentation between North American black and white populations.

The magnitude of choroidal thickening by the inflammatory infiltrate, the intensity of tissue eosinophilia, and the proportion of the epithelioid cell response in the choroid compared to the lymphocytic infiltration were rated by a single observer who was 1 of the 3 independent observers among whom there was essential agreement in all cases in the prior study. ${ }^{5}$

The choroidal inflammatory reaction was rated mild if the greatest thickness of the postequatorial choroid did not exceed half of a 'high-dry' field $(430 \times)$, moderate if the choroidal thickness exceeded one-half but was less than 1 complete high-dry field, and marked if the thickness of the postequatorial choroidal inflammation exceeded 1 full high-dry field.

The epithelioid cell response was evaluated by comparing the relative proportion of choroidal thickening accounted for by the nests of epithelioid cells compared to the lymphocytic infiltrate. Eosinophils were considered to be increased if 3 or more were seen in the choroid in 5 representative high-dry fields. This increase was considered heavy if more than 20 eosinophils were seen in a high-power field.

\section{Results}

The 2 groups of cases of sympathetic ophthalmia were chosen because they appeared to represent degrees of pigmentation intermediate between the white and black patients. The histopathological features of sympathetic ophthalmia that seem to be associated with pigment were observed to occupy 
an intermediate position with respect to the severity of the inflammation, the epithelioid response, and the degree of eosinophilia (Table 1). Although increased eosinophilia is associated with increased pigmentation (Table 2), this association may be fortuitous and simply reflect the observation that eosinophilia is a function of the severity of disease in all groups studied (Table 3). The epithelioid response appeared to be more closely related pigmentation. In severe cases in whites the epithelioid response is not as predominant as in black patients, though the degree of epithelioid response is difficult to quantitate.

\section{Discussion}

Our observations confirm the previously described association between degree of pigmentation and the intensity of uveal inflammation in sympathetic

Table 1 Degree of inflammatory thickening of the choroid in sympathetic ophthalmia

\begin{tabular}{|c|c|c|c|c|}
\hline \multirow{2}{*}{ Pigmentation } & \multirow{2}{*}{$\begin{array}{l}\text { No. of } \\
\text { patients }\end{array}$} & \multicolumn{3}{|c|}{ Choroidal thickening (per cent) } \\
\hline & & Mild & moderate & Severe \\
\hline Caucasians & 218 & 60 & 24 & 16 \\
\hline Mixed group & 19 & 21 & 37 & 42 \\
\hline Japanese & 25 & 24 & 28 & 48 \\
\hline Black & 34 & 11 & 29 & 60 \\
\hline
\end{tabular}

Table 2 Eosinophilia in the choroid of sympathetic ophthalmia

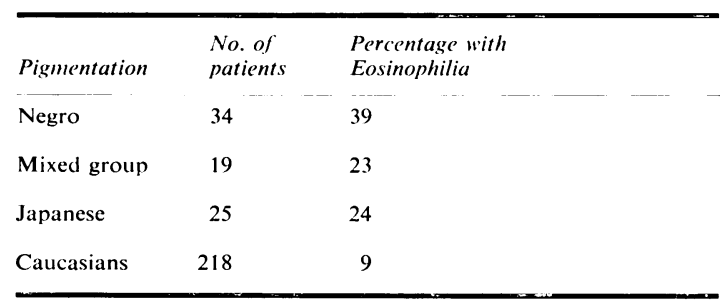

Table 3 Eosinophilia as a function of inflammation

\begin{tabular}{lccl}
\hline Eosinophilia* & Mild (\%) & Moderate (\%) & Severe (\%) \\
\cline { 2 - 4 } Negroes (13) & 15 & 15 & 70 \\
Japanese (7) & 0 & 43 & 57 \\
Mixed (5) & 20 & 20 & 60 \\
Caucasian (19) & 10 & 15 & 75 \\
\hline
\end{tabular}

* Three or more eosinophils in the choroid in 5 representative high-dry fields. ophthalmia. As predicted, patients with pigmentation intermediate between black and white patients have a form of sympathetic ophthalmia showing intermediate intensities of inflammatory reaction.

Fuchs originally suggested that the pigment containing epithelioid cells in sympathetic ophthalmia may represent modified uveal melanocytes. ${ }^{?}$ This possibility has been discussed in some detail by Hogan and Zimmerman, who point out ${ }^{8}$ that there is insufficient tissue necrosis in sympathetic ophthalmia to account for the amount of apparent pigment 'phacocytosis'.

Current evidence suggests an alternative to the belief that the pigment-containing epithelioid cells have phagocytosed the alleged offending uveal pigment. The hypothesis that pigment-containing epithelioid cells represent modified uveal melanocytes is supported by several studies. Various intermediate stages between melanocytes and the socalled pigment-containing epithelioid cells have been observed. ${ }^{910}$ Autophagy of melanosomes by pigment-containing cells has been described. ${ }^{11}$ There has been a detailed demonstration that the epithelioid cells of Dalen-Fuchs nodules are modified retinal pigment epithelial cells. ${ }^{12}{ }^{13}$

A recent observation on the association of an experimental form of sympathetic ophthalmia with inflammation of the pineal gland suggests that a disturbance in melantonin metabolism may provide a link between the uveal inflammation and pigmentary disturbances. ${ }^{14}$

The association of eosinophilia with pigmentation appears to be fortuitous, as there are no known mechanisms of association. This appears to be a reflection of the correlation between eosinophilia and the severity of the inflammation. The association of eosinophilia with severe inflammation and the relative concentration of eosinophils in the inner choroid have important immunological implications. In experimental sympathetic ophthalmia eosinophils occur only in the more severe forms of disease, are associated an increase in antibodies to the sympathogenic antigen, and may be suppressed by the C 3 inactivator factor of cobra venom (which depletes complement and interferes with immune complex disease). These observations suggest that an immune complex mechanism of pathogenesis may participate in very severe forms of disease, though the basic pathogenic mechanism appears to involve cell mediated hypersensitivity. ${ }^{15}$

The preferential localisation of eosinophils in the inner choroid in both human and experimental sympathetic ophthalmia may be a reflection of the site at which the immunological surveillance system first encounters the sympathogenic antigen, which is presumably released from the immunologically 
privileged site between the middle limiting membrane of the retina and Bruch's membrane as a result of the digestion of outer segment containing phagosomes by the retinal pigment epithelium. ${ }^{34}$

Our understanding of the pathogenesis of the pigment-containing epithelioid cells in sympathetic ophthalmia is uncertain. Recent evidence suggests that the hypothesis that the pigment-containing epithelioid cells in sympathetic ophthalmia represent modified uveal melanocytes may be considered as an alternative to the opinion that these cells have phagocytosed the pigment. These alternatives can be tested when reliable specific surface markers are developed for macrophages and melanocytes.

The authors are indebted to Professor L. E. Zimmerman for his thoughtful advice.

Supported by research grant EY-00887 from the National Eye Institute, National Institutes of Health, Bethesda, Maryland.

\section{References}

${ }^{1}$ Elsching A. Studies in sympathetic ophthalmia II. The antigenic effect of eye pigments, Albrecht von Graefes Arch Klin Ophthalmol 1910;76:509-46.

${ }^{2}$ Elsching A, Salus R. Studies on Sympathetic Ophthalmia IV. The antigenic effects of uveal pigments, Albrecht von Graefes Arch Klin Ophthalmol 1911;79:428-441.

${ }^{3}$ Marak GE. Immunopathology of Sympathetic Ophthalmia, Mod Probl Ophthalmol 1976;16:102-105.
${ }^{4}$ Wacker WB, Rao NA, Marak GE. Experimental Sympathetic Ophthalmia. In: Silverstein A, O'Connor R, eds. Immunology and Immunopathology of the Eye. New York: Masson, 1979.

${ }^{5}$ Marak GE, Font RL, Zimmerman LE. Histologic variations related to race in sympathetic ophthalmia. Am J Ophthalmol 1974;78:935-38.

'Easom HA, Zimmerman LE. Sympathetic ophthalmia and bilateral phacoanaphylaxis. Arch Ophthalmol (1964);72: 9-15.

${ }^{7}$ Fuchs T. Concerning sympathetic ophthalmia (including remarks about serous traumatic iritis). Albrecht von Graefes Arch Klin Ophthalmol 1905;11:365-456.

${ }^{8}$ Hogan MJ, Zimmerman LE. Ophthalmic Pathology. Philadelphia: Saunders, 1962.

'Ikui H, Kimura K, Iwaki S. Electron microscopic study of ultrathin sections of sympathetic ophthalmia. Jpn J Ophthalmol 1958;2:13-20.

${ }^{10}$ Ikui H, Kimura K, Nishio T. Further studies on sympathetic ophthalmia with electron microscope, Jpn J Ophthalmol $1959 ; 3: 27-37$.

${ }^{11}$ Mixon R, Feeney L. A melanotic retinal pigment epithelium: the role of enzymes in the biology of melanin. Invest Ophthal Visual Sci 1978; 17 suppl., 115.

${ }^{12}$ Ishikawa $T$, Ikui $H$. The fine structure of the DalenFuchs nodule in sympathetic ophthalmia, Jpn J Ophthalmol $1972 ; 16: 254-65$.

${ }^{13}$ Font RL, Fine BS. (In preparation). Electron Microscopy of Dalen-Fuchs nodules in sympathetic ophthalmia.

${ }^{14}$ Kalsow CM, Wacker WB. The Pineal Gland and Experimental Allergic Uveitis. In: Silverstein A, O'Connor R, eds. Immunology and Immunopathology of the Eye. New York: Masson, 1979.

${ }^{15}$ Rao NA, Wacker WB, Marak GE. Experimental allergic uveitis, clinicopathologic features associated with varying doses of S antigen. Arch Ophthalmol 1979:97:1954-8. 